\title{
CONSTRUCTION OF THREEFOLDS WITH FINITE CANONICAL MAP*
}

\author{
G. CASNATI \\ Dipartimento di Matematica, Politecnico di Torino, c.so Duca degli Abruzzi 24, 10129 Torino, Italy \\ e-mail: casnati@calvino.polito.it \\ and P. SUPINO \\ Dipartimento di Matematica "Vito Volterra", Università degli Studi di Ancona, via Brecce Bianche, \\ 60131 Ancona, Italy \\ e-mail: supino@popcsi.unian.it
}

(Received 24 March, 2000; accepted 8 September 2000)

\begin{abstract}
We study the moduli space of pluriregular threefolds of general type with $K_{X}^{3}=8,10,12$ and $p_{g}(X)=5$ and whose canonical map is a finite morphism onto a smooth quadric $Q \subseteq \mathbb{P}_{\mathbb{C}}^{4}$.
\end{abstract}

2000 Mathematics Subject Classification. 14J30, 14E20, 14 J15.

1. Introduction. The classical method of classification of surfaces of general type goes through the study of the canonical map. Although the extension of this approach to higher dimensions fails to fill up the classification of varieties by means of their birational invariants, a partial investigation may be done. In particular, one can study the case in which the canonical map is generically finite.

If $X$ is any algebraic $n$-fold of general type over the complex field $\mathbb{C}$ whose canonical sheaf is nef and whose canonical map $\varphi$ is generically finite of degree $d$, then $K_{X}^{n} \geq d\left(p_{g}(X)-n\right)$. In [14] it is proved that if equality holds and $\left|K_{X}\right|$ has no fixed components then it is base point free. In particular $\varphi$ is a morphism whose image is a variety of minimal degree. If there are no exceptional curves, then $\varphi$ is also flat and finite. Briefly, we call $\varphi$ a cover of degree $d$.

In this paper, we apply the theory of covers of degree $d$, developed in [6], [4] and [5] to the case $n=3$ and $d=4,5,6$. (See [14] for the case $n=d=3$, [10] for the case $n=d=2$ and [4] for the case $n=2$ and $p_{g}(X)=3$.) In particular we prove the following result.

THEOREM. There exists an explicit construction for smooth, minimal, pluriregular (this means $\left.h^{1}\left(X, \mathcal{O}_{X}\right)=h^{2}\left(X, \mathcal{O}_{X}\right)=0\right)$, threefolds of general type $X$ with $p_{g}(X)=5$, $K_{X}^{3}=2 d=8,10,12$, whose canonical map is a cover $\varrho: X \rightarrow Q$ of degree $d$ onto a smooth quadric $Q \subseteq \mathbb{P}_{\mathbb{C}}^{4}$.

In the cases $K_{X}^{3}=8,10$ (resp. $K_{X}^{3}=12$ ) this is (resp. is not) the only possible way to obtain every threefold with those invariants and with such a canonical map.

$\overline{\text { *This work }}$ was done in the framework of the SCIENCE contract SCI-0398-C(A). 
Moreover, in the case $d=4$, we also give a rough description of the locus $\mathfrak{M}_{8,5}^{\text {quadric }}$ of such kind of threefolds, inside the moduli space $\mathfrak{M}_{8,5}$ of smooth, pluriregular threefolds of general type $X$ with $p_{g}(X)=5, K_{X}^{3}=8$. (See Section 5 for the existence of $\mathfrak{M}_{8,5}$.) More precisely we prove the following result.

$=$ THEOREM. $\mathfrak{M}_{8,5}^{q u a d r i c}$ is unirational of dimension 126. The unique component of $\mathfrak{M}_{8,5}$ containing $\mathfrak{M}_{8,5}^{\text {quadric }}$ has dimension 128.

The general deformation of each threefold corresponding to a point of $\mathfrak{M}_{8,5}^{\text {quadric }}$ has birational canonical morphism onto a hypersurface of degree 8 of $\mathbb{P}_{\mathbb{C}}^{4}$ with ordinary singularities.

In Section 6 we give some partial results about covers of degree $d=5,6$ of smooth quadrics, whereas in the last section we give some examples of threefolds of general type $X$ with $h^{1}\left(X, \mathcal{O}_{X}\right)=h^{2}\left(X, \mathcal{O}_{X}\right)=0, K_{X}^{3}=d\left(p_{g}(X)-3\right)$ and odd $p_{g}(X) \geq 7$, as covers of threefolds of minimal degree in $\mathbb{P}_{\mathbb{C}}^{p_{g}(X)-1}$.

2. A result on covers. The aim of the following section is to list some results regarding covers which will be helpful in the following sections (see [6], [4], [5]). We begin by recalling the definition of cover. (See [9].)

Definition 2.1. Let $Y$ be an integral scheme i.e. a noetherian scheme which is separated and of finite type over $\mathbb{C}$. Let $X$ be another scheme with $\operatorname{dim}(X)=\operatorname{dim}(Y)$. A cover $\varrho: X \rightarrow Y$ is a flat and infinite morphism. Its degree $\operatorname{deg}(\varrho)$ is the rank of the locally free $\mathcal{O}_{Y}$-sheaf $\varrho_{*} \mathcal{O}_{X}$.

If $Y$ is smooth and $X$ is Cohen-Macaulay, then every finite surjective morphism is a cover.

If $\varrho: X \rightarrow Y$ is a cover of degree $d$, then we have the trace map $\varrho^{\#}: \mathcal{O}_{Y} \rightarrow \varrho_{*} \mathcal{O}_{X}$. The locally free $\mathcal{O}_{Y}$-sheaf $\breve{\mathcal{E}}=$ coker $\varrho^{\#}$ is called the Tschirnhausen module of $\varrho$ (see [11]) and we have $\varrho_{*} \mathcal{O}_{X} \cong \mathcal{O}_{Y} \oplus \check{\mathcal{E}}$. (Generalize the proof of Lemma 2.2 in [11].)

A cover $\varrho: X \rightarrow Y$ is called Gorenstein if the scheme-theoretic fibre $\varrho^{-1}(y)$ is Gorenstein for every $y \in Y$ : this is equivalent to the invertibility of the relative dualizing sheaf $\omega_{X \mid Y}$. If $Y$ is Gorenstein, then $\varrho$ is Gorenstein if and only if $X$ is also Gorenstein. Relative duality yields $\varrho_{*} \omega_{X \mid Y} \cong \mathcal{O}_{Y} \oplus \mathcal{E}$.

Let $\varrho: X \rightarrow Y$ be a Gorenstein cover of degree $d$ with $Y$ integral and Tschirnhausen module $\breve{\mathcal{E}}$. It is shown in [6] that $\varrho$ factors through an embedding $i: X \hookrightarrow \mathbb{P}:=\mathbb{P}(\mathcal{E})$ followed by the canonical projection $\pi: \mathbb{P} \rightarrow Y$.

In any case $X$ is the zero locus $D_{0}(\delta)$ of a section $\delta \in H^{0}\left(\mathbb{P}, \pi^{*} \check{\mathcal{F}}(2)\right)$, where $\mathcal{F}$ is a locally free $\mathcal{O}_{Y}$-sheaf of rank $N:=d(d-3) / 2$. $\mathcal{E}$ and $\mathcal{F}$ are called the invariants of $\varrho$.

If $\varphi: \mathcal{U} \rightarrow \mathcal{V}$ is a morphism between locally free $\mathcal{O}_{Y}$-sheaves, $D_{r}(\varphi) \subseteq Y$ denotes the subscheme locally defined by the vanishing of the $(r+1) \times(r+1)$-minors of a local matrix of $\varphi$.

Let $\varrho: X \rightarrow Y$ be a Gorenstein cover of degree $d \geq 3$, where both $X$ and $Y$ are Gorenstein. Since $\omega_{X} \cong \omega_{X \mid Y} \otimes \varrho^{*} \omega_{Y}$ the following proposition allows us to choose the invariants $\mathcal{E}$ and $\mathcal{F}$ so as to ensure that $\varrho$ is the canonical map.

Proposition 2.2. Let both $X$ and $Y$ be integral, $\varrho: X \rightarrow Y$ be a Gorenstein cover of degree $d \geq 3, \mathcal{L} \in \operatorname{Pic}(Y)$ and assume that $\mathcal{E}:=\mathcal{L}^{\oplus d-2} \oplus \mathcal{L}^{2}$. 
The following assertions are equivalent:

(i) $\mathcal{F}:=\left(\mathcal{L}^{2}\right)^{\oplus N}, N:=d(d-3) / 2$;

(ii) $\omega_{X \mid Y} \cong \varrho^{*} \mathcal{L}^{2}$.

Proof. Choose linearly independent sections $u_{1}, \ldots, u_{d-2} \in H^{0}\left(Y, \mathcal{E} \otimes \mathcal{L}^{-1}\right) \cong$ $H^{0}\left(\mathbb{P}, \mathcal{O}_{\mathbb{P}}(1) \otimes \pi^{*} \mathcal{L}^{-1}\right)$ and $u_{d-1} \in H^{0}\left(Y, \mathcal{E} \otimes \mathcal{L}^{-2}\right) \cong H^{0}\left(\mathbb{P}, \mathcal{O}_{\mathbb{P}}(1) \otimes \pi^{*} \mathcal{L}^{-2}\right)$, where $\mathbb{P}=\mathbb{P}(\mathcal{E})$. Then each section $\delta \in H^{0}\left(\mathbb{P}, \pi^{*} \breve{\mathcal{F}}(2)\right)$ decomposes as

$$
\delta=\sum_{i, j=1}^{d-2} a_{i, j} u_{i} u_{j}+2 \sum_{i=1}^{d-2} b_{i} u_{i} u_{d-1}+c u_{d-1}^{2},
$$

where $a_{i, j} \in H^{0}\left(Y, \check{\mathcal{F}} \otimes \mathcal{L}^{2}\right), b_{i} \in H^{0}\left(Y, \check{\mathcal{F}} \otimes \mathcal{L}^{3}\right), c \in H^{0}\left(Y, \check{\mathcal{F}} \otimes \mathcal{L}^{4}\right)$

If (i) holds, then the splitting type of $\mathcal{F}$ yields $a_{i, j} \in H^{0}\left(Y, \mathcal{O}_{Y}\right)^{N}, b_{i} \in H^{0}(Y, \mathcal{L})^{N}$, $c \in H^{0}\left(Y, \mathcal{L}^{2}\right)^{N}$. In particular, $X \subseteq \mathbb{P}$ is the scheme-theoretic intersection of the $N$ hypersurfaces $Q_{h}$ of equations

$$
q_{h}(u):=\sum_{i, j=1}^{d-2} a_{i, j}^{(h)} u_{i} u_{j}+2 \sum_{i=1}^{d-2} b_{i}^{(h)} u_{i} u_{d-1}+c^{(h)} u_{d-1}^{2},
$$

where $a_{i, j}^{(h)} \in H^{0}\left(Y, \mathcal{O}_{Y}\right), b_{i}^{(h)} \in H^{0}(Y, \mathcal{L}), c^{(h)} \in H^{0}\left(Y, \mathcal{L}^{2}\right)$. If $U:=\left\{u_{d-1}=0\right\}$, then $X \cap U$ is defined in $U$ by the equations

$$
\widehat{q_{h}}(u):=\sum_{i, j=1}^{d-2} a_{i, j}^{(h)} u_{i} u_{j},
$$

which do not depend on the point $y \in Y$. Since $U \nsubseteq X$, there is $y \in Y$ such that $\varrho^{-1}(y) \cap U=\emptyset$ and hence $X \cap U=\emptyset$. It follows that $\mathcal{O}_{X} \cong \mathcal{O}_{\mathbb{P}}(U)_{\mid X} \cong$ $\left(\mathcal{O}_{\mathbb{P}}(1) \otimes \pi^{*} \mathcal{L}^{-2}\right)_{\mid X} \cong \omega_{X \mid Y} \otimes \varrho^{*} \mathcal{L}^{-2}$, i.e. $\omega_{X \mid Y} \cong \varrho^{*} \mathcal{L}^{2}$.

Conversely assume (ii). By Proposition 5.1 of [6], we have the exact sequence

$$
0 \longrightarrow \mathcal{F} \longrightarrow \mathcal{S}^{2} \mathcal{E} \stackrel{\psi}{\longrightarrow} \varrho_{*} \omega_{X \mid Y}^{2} \longrightarrow 0,
$$

where $\varrho_{*} \omega_{X \mid Y}^{2} \cong \mathcal{L}^{2} \oplus\left(\mathcal{L}^{3}\right)^{\oplus d-2} \oplus \mathcal{L}^{4}$ and $\mathcal{S}^{2} \mathcal{E} \cong\left(\mathcal{L}^{2}\right)^{\oplus m} \oplus\left(\mathcal{L}^{3}\right)^{\oplus d-2} \oplus \mathcal{L}^{4}\left(m:=\left(\begin{array}{c}d-1 \\ 2\end{array}\right)\right)$. The matrix of $\psi$ is

$$
M_{\psi}:=\left(\begin{array}{ccc}
a & 0 & 0 \\
D & B & 0 \\
e & f & c
\end{array}\right)
$$

where $a=\left(a_{1}, \ldots, a_{m}\right), D=\left(d_{i, j}\right)_{i=1, \ldots, d-2, j=1, \ldots, m}, e=\left(e_{1}, \ldots, e_{m}\right), B=\left(b_{i, j}\right)_{i, j=1, \ldots, d-2}$, $f=\left(f_{1}, \ldots, f_{d-2}\right)$ and $a_{i}, b_{i, j}, c \in H^{0}\left(Y, \mathcal{O}_{Y}\right), d_{i, j}, f_{i} \in H^{0}(Y, \mathcal{L})$ and $e_{i} \in H^{0}\left(Y, \mathcal{L}^{2}\right)$.

Since $\mathrm{rk} \psi=d$, we have $a \neq 0$. Let

$$
\operatorname{rk}\left(\begin{array}{cc}
B & 0 \\
f & c
\end{array}\right) \leq d-2
$$


Then the system $M_{\psi} x=0$ has a non-trivial solution $x:=\left(0, \ldots, 0, \alpha_{1}, \ldots, \alpha_{d-2}, q\right)$, where $\alpha_{i} \in \mathbb{C}, q \in H^{0}(Y, \mathcal{L})$; hence $X \subseteq Q$, where $Q \subseteq \mathbb{P}:=\mathbb{P}(\mathcal{E})$ is the subscheme defined by the global equation $u_{0}\left(\sum_{i=1}^{d-1} \alpha_{i} u_{i}+q u_{0}\right)=0$.

If $\left(\alpha_{1}, \ldots, \alpha_{d-2}\right) \neq 0$, then there are distinct subbundles $\mathbb{P}_{i} \subseteq \mathbb{P}(i=1,2)$ such that $Q=\mathbb{P}_{1} \cup \mathbb{P}_{2}$. Hence $X=\left(X \cap \mathbb{P}_{1}\right) \cup\left(X \cap \mathbb{P}_{2}\right)$. Since $X \cap \mathbb{P}_{i}$ is an arithmetically Gorenstein subscheme of $\mathbb{P}_{\mathbb{C}}^{d-2}$, its ideal is generated by quadrics so that $X \nsubseteq \mathbb{P}_{i}$ and $X$ would be reducible.

If $\left(\alpha_{1}, \ldots, \alpha_{d-2}\right)=0$ then $q \neq 0$ : hence the reduced scheme $(X)_{\text {red }} \subseteq\left\{u_{0}=0\right\}$, which is absurd since $\varrho$ is generically smooth, so that

$$
\operatorname{rk}\left(\begin{array}{cc}
B & 0 \\
f & c
\end{array}\right)=d-1
$$

Hence we may consider the morphism $\varphi: \varrho_{*} \omega_{X \mid Y}^{2} \rightarrow \mathcal{S}^{2} \mathcal{E}$ associated to the matrix

$$
M_{\varphi}:=\left(\begin{array}{ccc}
a^{\prime} & 0 & 0 \\
P & B^{-1} & 0 \\
Q & R & c^{-1}
\end{array}\right),
$$

where $a^{\prime}={ }^{t}(0, \ldots, 0,1)$ and $P, Q, R$ are matrices uniquely determined by the conditions $D a^{\prime}+B P=e a^{\prime}+f P+c Q=f B^{-1}+c R=0$. Since $M_{\psi} \cdot M_{\varphi}=I$, it follows that $\mathcal{F} \cong \mathcal{S}^{2} \mathcal{E} / \operatorname{im}(\varphi) \cong\left(\mathcal{L}^{2}\right)^{\oplus N}$, where $N:=m-d=d(d-3) / 2$.

3. Existence results in dimension three. From now on let $\mathcal{L} \in \operatorname{Pic}(Y)$, $\mathcal{E}:=\mathcal{L}^{\oplus d-2} \oplus \mathcal{L}^{2}, \mathbb{P}:=\mathbb{P}(\mathcal{E})$ and $\mathcal{F}:=\left(\mathcal{L}^{2}\right)^{\oplus N}, N:=d(d-3) / 2$. We list some Bertini theorems which will be helpful in the following sections.

Proposition 3.1. Let $Y$ be an integral threefold, $d=4, \mathcal{L}, \mathcal{E}$ and $\mathcal{F}$ be as above. The general section $\delta$ of $\pi^{*} \breve{\mathcal{F}}(2)$ defines a Gorenstein cover $\varrho: X:=D_{0}(\delta) \rightarrow Y$ of degree 4 with invariants $\mathcal{E}$ and $\mathcal{F}$.

If $Y$ is smooth and projective and $\mathcal{L}$ is globally generated, then $X$ is smooth and connected.

Proof. The statement is a particular case of Theorem 4.5 of [6].

If $d \geq 5$ the general section $\delta \in H^{0}\left(\mathbb{P}, \pi^{*} \breve{\mathcal{F}}(2)\right)$ cannot define a cover of degree $d$, since the codimension of $D_{0}(\delta)$ inside $\mathbb{P}$ is $N>d-2$. In [4] it is proved that for each Gorenstein cover $\varrho: X \rightarrow Y$ of degree $d=5$ with invariants $\mathcal{E}$ and $\mathcal{F}$, there exists a skew-symmetric map $\delta: \pi^{*} \mathcal{F}(-1) \rightarrow \pi^{*} \breve{\mathcal{F}} \otimes \operatorname{det} \mathcal{E}^{-1}$ whose pfaffian locus inside $\mathbb{P}$ (i.e. the subscheme locally defined by the $4 \times 4$-pfaffians of a local matrix of $\delta$ ) coincides with $X$. We have the following result.

Proposition 3.2. Let $Y$ be an integral threefold, $d=5, \mathcal{L}, \mathcal{E}$ and $\mathcal{F}$ be as above. The general skew-symmetric map $\delta: \pi^{*} \mathcal{F}(-1) \rightarrow \pi^{*} \breve{\mathcal{F}} \otimes \operatorname{det} \mathcal{E}^{-1}$ defines a Gorenstein cover $\varrho: X \rightarrow Y$ of degree 5 with invariants $\mathcal{E}$ and $\mathcal{F}$.

If $Y$ is smooth and projective and $\mathcal{L}$ is globally generated, then $X$ is smooth and connected. 
Proof. Choose independent sections $u, v, w \in H^{0}\left(Y, \mathcal{E} \otimes \mathcal{L}^{-1}\right) \cong H^{0}\left(\mathbb{P}, \mathcal{O}_{\mathbb{P}}(1) \otimes\right.$ $\left.\pi^{*} \mathcal{L}^{-1}\right)$ and $t \in H^{0}\left(Y, \mathcal{E} \otimes \mathcal{L}^{-2}\right) \cong H^{0}\left(\mathbb{P}, \mathcal{O}_{\mathbb{P}}(1) \otimes \pi^{*} \mathcal{L}^{-2}\right)$.

There is a decomposition $\delta=\delta_{t} t+\delta_{u} u+\delta_{v} v+\delta_{w} w$, where $\delta_{u}, \delta_{v}$ and $\delta_{w}$ (resp. $\delta_{t}$ ) are $5 \times 5$ alternating matrices whose entries belong to $H^{0}\left(Y, \mathcal{O}_{Y}\right)$ (resp. to $H^{0}(Y, \mathcal{L})$ ). Let $P f_{4}(\delta)$ be the ideal generated by the $4 \times 4$ pfaffians of a matrix of $\delta$ and set $X:=V_{+}\left(P f_{4}(\delta)\right.$ ) (for each ideal $I, V_{+}(I)$ denotes the variety associated to $I$ ).

Let $\delta^{\prime}:=\delta_{u} u+\delta_{v} v+\delta_{w} w$ and $T:=\{t=0\}$. If $\delta^{\prime} \neq 0$, then there is $y \in \mathbb{P}_{\mathbb{C}}^{2}$ such that $\mathbb{P}_{y} \cap X \cap T=\emptyset$. Hence $\mathbb{P}_{y} \cap X$ is Gorenstein by [3]. It follows that $P f_{4}\left(\delta^{\prime}\right)$ is a homogeneous, Gorenstein ideal and $\mathbb{C}[u, v, w] / P f_{4}\left(\delta^{\prime}\right)$ is local and artinian. Since $P f_{4}\left(\delta^{\prime}\right)$ does not depend on $y$ it follows that $X$ is fibrewise Gorenstein and $X \cap T=\emptyset$. In particular $\operatorname{dim}\left(X \cap \mathbb{P}_{y}\right)=0$ and $\operatorname{deg}\left(X \cap \mathbb{P}_{y}\right)=\operatorname{dim}_{\mathbb{C}}\left(\mathbb{C}[u, v, w] / P f_{4}\left(\delta^{\prime}\right)\right)$ is constant. Hence $\varrho:=\pi_{\mid X}$ is a cover. $\mathcal{O}_{X}$ has a resolution of the form

$$
\begin{aligned}
0 \rightarrow \mathcal{O}_{\mathbb{P}}(-5) \otimes \pi^{*} \mathcal{L}^{5} & \rightarrow\left(\mathcal{O}_{\mathbb{P}}(-3) \otimes \pi^{*} \mathcal{L}^{3}\right)^{\oplus 5} \stackrel{\delta}{\rightarrow} \\
& \rightarrow\left(\mathcal{O}_{\mathbb{P}}(-2) \otimes \pi^{*} \mathcal{L}^{2}\right)^{\oplus 5} \rightarrow \mathcal{O}_{\mathbb{P}} \rightarrow \mathcal{O}_{X} \rightarrow 0 .
\end{aligned}
$$

Thus the invariants of $\varrho$ are $\mathcal{E}, \mathcal{F}$ (by Theorem 2.1 of [6])) and $\operatorname{deg} \varrho=5$ (by (3.2.1) and direct computation).

If $\mathcal{L}$ is globally generated, then $H^{0}(Y, \check{\mathcal{E}})=0$ and $V_{+}\left(P f_{4}(\delta)\right)$ is non-singular in codimension 6. (See Section 6 of [3].)

If $d \geq 6$ a description of covers of degree $d$ is missing. In [5] a particular kind of cover of degree $d=6$ called scandinavian is defined. Take two locally free $\mathcal{O}_{Y^{-}}$ sheaves $\mathcal{A}$ and $\mathcal{B}$, both of rank 3 . For each map $\delta: \pi^{*} \mathcal{A}(-1) \rightarrow \pi^{*} \mathcal{B}$ we can consider the locus $X:=D_{1}(\delta) \subseteq \mathbb{P}$. If $\operatorname{dim}(Y) \leq 2$, it is proved in [5] that $\varrho:=\pi_{\mid X}: X \rightarrow Y$ is a cover of degree 6 with invariants $\mathcal{E}$ and $\mathcal{F}:=\mathcal{S}^{2} \mathcal{A} \otimes \mathcal{S}^{2} \mathcal{B}$ under suitable hypotheses on $Y$ and the sheaves $\mathcal{A}, \mathcal{B}$ and $\mathcal{E}$. In the case $\operatorname{dim}(Y)=3$ we have the following result.

Proposition 3.3. Let $Y$ be an integral threefold, $d=6$, and $\mathcal{L}, \mathcal{E}, \mathcal{F}$ be as above. Define $\mathcal{A}:=\mathcal{O}_{Y}{ }^{\oplus 3}, \mathcal{B}:=\left(\mathcal{L}^{-1}\right)^{\oplus 3}$. The general map $\delta: \pi^{*} \mathcal{A}(-1) \rightarrow \pi^{*} \mathcal{B}$ defines a scandinavian cover $\varrho: X \rightarrow Y$ of degree 6 with invariants $\mathcal{E}$ and $\mathcal{F}$.

If $Y$ is smooth and projective and $\mathcal{L}$ is globally generated, then $X$ is smooth and connected.

Proof. We choose independent sections $t, u, v, w \in H^{0}\left(Y, \mathcal{E} \otimes \mathcal{L}^{-1}\right) \cong$ $H^{0}\left(\mathbb{P}, \mathcal{O}_{\mathbb{P}} \otimes \pi^{*} \mathcal{L}^{-1}\right)$ and $s \in H^{0}\left(Y, \mathcal{E} \otimes \mathcal{L}^{-2}\right) \cong H^{0}\left(\mathbb{P}, \mathcal{O}_{\mathbb{P}} \otimes \pi^{*} \mathcal{L}^{-2}\right)$.

There is a decomposition $\delta=\delta_{s} s+\delta_{t} t+\delta_{u} u+\delta_{v} v+\delta_{w} w$, where $\delta_{t}, \delta_{u}, \delta_{v}, \delta_{w}$ (resp. $\delta_{s}$ ) are defined by $3 \times 3$ matrices whose entries are in $H^{0}\left(Y, \mathcal{O}_{Y}\right)$ (resp. $H^{0}(Y, \mathcal{L})$ ). Let $X:=D_{1}(\delta)$.

Let $\delta^{\prime}:=\delta_{t} t+\delta_{u} u+\delta_{v} v+\delta_{w} w$ and $S:=\{s=0\}$. If $\delta^{\prime} \neq 0$, as in the proof of the previous proposition there is $y \in Y$ such that $X \cap \mathbb{P}_{y}$ is Gorenstein. (See [8].) Moreover the ideal $I_{2}\left(\delta^{\prime}\right)$ generated by the $2 \times 2$-minors of a matrix of $\delta^{\prime}$ is homogeneous and Gorenstein. Also $\mathbb{C}[t, u, v, w] / I_{2}\left(\delta^{\prime}\right)$ is local and artinian, and $\operatorname{dim}\left(X \cap \mathbb{P}_{y}\right)=0, \operatorname{deg}\left(X \cap \mathbb{P}_{y}\right)=\operatorname{dim}_{\mathbb{C}}\left(\mathbb{C}[t, u, v, w] / I_{2}\left(\delta^{\prime}\right)\right)$ is constant, so that $\varrho:=\pi_{\mid X}$ is a cover. $\mathcal{O}_{X}$ has a resolution as an $\mathcal{O}_{\mathbb{P}}$-module of the form

$$
\begin{aligned}
0 & \rightarrow \mathcal{O}_{\mathbb{P}}(-6) \otimes \pi^{*} \mathcal{L}^{6} \rightarrow\left(\mathcal{O}_{\mathbb{P}}(-4) \otimes \pi^{*} \mathcal{L}^{4}\right)^{\oplus 9} \rightarrow \\
& \rightarrow\left(\mathcal{O}_{\mathbb{P}}(-3) \otimes \pi^{*} \mathcal{L}^{3}\right)^{\oplus 16} \rightarrow\left(\mathcal{O}_{\mathbb{P}}(-2) \otimes \pi^{*} \mathcal{L}^{2}\right)^{\oplus 9} \rightarrow \mathcal{O}_{\mathbb{P}} \rightarrow \mathcal{O}_{X} \rightarrow 0 .
\end{aligned}
$$


(See [8].) Hence the invariants of $\varrho$ are $\mathcal{E}, \mathcal{F}$, by Theorem 2.1 of [6], and $\operatorname{deg} \varrho=6$, by (3.3.1) and direct computation.

If $\mathcal{L}$ is globally generated, then $H^{0}(Y, \breve{\mathcal{E}})=0$ and $D_{1}(\delta)$ is non-singular in codimension 8 .

4. Pluriregular threefolds with $\boldsymbol{p}_{\mathbf{g}}=\mathbf{5}$. Let $X$ be a smooth threefold. Recall that the Riemann-Roch theorem for $2 K_{X}$ implies that $K_{X}^{3}=6 \chi\left(\mathcal{O}_{X}\right)+2 \chi\left(\omega_{X}^{2}\right)$; thus, in any case, $K_{X}^{3}$ is even, say $K_{X}^{3}=2 d$.

Assume $p_{g}(X)=5$ and that $X$ is pluriregular; i.e. $h^{1}\left(X, \mathcal{O}_{X}\right)=h^{2}\left(X, \mathcal{O}_{X}\right)=0$. If the canonical map $\varphi_{X}: X \rightarrow \mathbb{P}_{\mathbb{C}}^{4}$ is finite, then $X$ lies in finitely many families. In particular if $d \geq 4$ (for the case $d=3$ see [14]) we have two interesting extremal cases; i.e. $X$ is either a double cover of a threefold of degree $d$ or a $d$-fold cover of a quadric. In this section we study this second case.

THEOREM 4.1. There exist smooth, pluriregular, threefolds of general type $X$ with $p_{g}(X)=5, K_{X}^{3}=2 d=8,10,12$, whose canonical map is cover $\varrho: X \rightarrow Q$ of degree $d$ onto a smooth quadric $Q \subseteq \mathbb{P}_{\mathbb{C}}^{4}$, with $\mathcal{E} \cong \mathcal{O}_{Q}(2)^{\oplus d-2} \oplus \mathcal{O}_{Q}(4)$.

Proof. Notice that $\mathcal{O}_{Q}(2)$ is globally generated and hence we may apply Propositions $3.1,3.2,3.3$ with $Y=Q$ and $\mathcal{L}:=\mathcal{O}_{Q}(2)$ in order to build covers $\varrho: X \rightarrow Q$. The canonical map of $X$ is $\varrho$ by Proposition 2.2.

In particular in the cases $d=4,5$ we can also prove the converse of the above theorem. Let $S$ be the spinor sheaf on $Q$. (See [12] for the definition and properties of $S$.) We begin by proving the following result.

Proposition 4.2. Let $X$ be a smooth, pluriregular threefold of general type with $p_{g}(X)=5, K_{X}^{3}=2 d$.

Assume that $\left|K_{X}\right|$ has no fixed part and that the canonical map is a cover $\varrho: X \rightarrow Q \subseteq \mathbb{P}_{\mathbb{C}}^{4}$ of degree d onto a smooth quadric.

Then there exists $p \geq 0$ such that $\mathcal{E} \cong S(2)^{p} \oplus S(3)^{p} \oplus \mathcal{O}_{Q}(2)^{d-2-4 p} \oplus \mathcal{O}_{Q}(4)$.

Proof. Since $\omega_{X}=\varrho^{*} \mathcal{O}_{Q}(1)$, we have $\omega_{X \mid Q}=\varrho^{*} \mathcal{O}_{Q}(4)$. Moreover the isomorphism

$$
\mathcal{O}_{Q}(-4) \oplus \mathcal{E}(-4)=\left(\varrho_{*} \omega_{X \mid Q}\right)(-4)=\varrho_{*} \mathcal{O}_{X}=\mathcal{O}_{Q} \oplus \check{\mathcal{E}}
$$

gives rise to a factorization of the identity on $\mathcal{O}_{Q}$ as $\mathcal{O}_{Q} \stackrel{i}{\longrightarrow} \mathcal{O}_{Q}(-4) \oplus \mathcal{E}(-4) \stackrel{r}{\longrightarrow}$ $\mathcal{O}_{Q}$. Since $h^{0}\left(Q, \mathcal{O}_{Q}(-4)\right)=0$, it follows that both $i$ and $r$ split through $\mathcal{E}(-4)$. Hence $\mathcal{O}_{Q}(4)$ is a direct summand of $\mathcal{E}$.

Since $\omega_{X} \cong \varrho^{*} \mathcal{O}_{Q}(1)$ is ample and $X$ is pluriregular one gets

$$
h^{i}\left(Q,\left(\varrho_{*} \check{\mathcal{E}}\right)(n)\right)=h^{i}\left(X,\left(\varrho_{*} \mathcal{O}_{X}\right)(n)\right)=h^{i}\left(X, \omega_{X}^{n}\right)=0 \quad(i=1,2)
$$

by the Kodaira vanishing theorem. It follows that Horrocks' theorem (see [1, Corollary 6.8]) gives us 


$$
\mathcal{E} \cong \bigoplus_{i=1}^{h} S\left(\beta_{i}\right) \oplus \bigoplus_{j=2 h+1}^{d-2} \mathcal{O}_{Q}\left(\alpha_{j}\right) \oplus \mathcal{O}_{Q}(4)
$$

where $S$ is the spinor sheaf on $Q$ (which is locally free of rank 2) and $\alpha_{j}, \beta_{i}$ are suitable integers.

In order to compute $\alpha_{j}$ and $\beta_{i}$, we fix a general hyperplane section $\bar{Q} \subseteq Q$. Then both $\bar{Q}$ and $\bar{X}:=\varrho^{-1}(\bar{Q})$ are smooth and connected and $\bar{\varrho}:=\varrho_{\mid} \mid \bar{X}$ is a cover of degree $d$. It follows that the dualized Tschirnhausen module of $\bar{\varrho}$ is $\overline{\mathcal{E}}:=\mathcal{E} \otimes \mathcal{O}_{\bar{Q}}$.

By adjunction on $X$ we obtain $\omega_{\bar{X}} \cong \bar{\varrho}^{*} \mathcal{O}_{\bar{Q}}(2)$. It follows that $h^{i}\left(\bar{X}, \bar{\varrho}^{*} \mathcal{O}_{\bar{Q}}(t)\right)=0$ for $t \geq 3$ and $i \geq 1$. Moreover $\chi\left(\mathcal{O}_{\bar{X}}\right)=\chi\left(\omega_{\bar{X}}\right)=\chi\left(\omega_{X}^{2}\right)-\chi\left(\omega_{X}\right)=d+8$ by RiemannRoch. It follows also from Riemann-Roch for $\overline{\varrho^{*}} \mathcal{O}_{\bar{Q}}(t)$ that

$$
h^{0}(\bar{Q}, \check{\mathcal{E}}(t))=d t(t-2)+d+8-(t+1)^{2}, \quad t \geq 3 .
$$

In Theorem 1.4 and Example 1.5 of [12] it is proved that $S \otimes \mathcal{O}_{\bar{Q}} \cong \mathcal{O}_{\bar{Q}}(-1,0) \oplus$ $\mathcal{O}_{\bar{Q}}(0,-1)$. Then formula (4.2.2) yields

$$
\overline{\mathcal{E}} \cong \bigoplus_{i=1}^{h}\left(\mathcal{O}_{\bar{Q}}\left(\beta_{i}-1, \beta_{i}\right) \oplus \mathcal{O}_{\bar{Q}}\left(\beta_{i}, \beta_{i}-1\right)\right) \oplus \bigoplus_{j=2 h+1}^{d-2} \mathcal{O}_{\bar{Q}}\left(\alpha_{j}\right) \oplus \mathcal{O}_{\bar{Q}}(4) .
$$

By (4.2.1), we get

$$
h^{0}\left(\bar{Q}, \mathcal{O}_{\bar{Q}}(-3) \oplus \overline{\mathcal{E}}(-3)\right)=h^{0}\left(\bar{Q}, \mathcal{O}_{\bar{Q}}(1) \oplus \check{\mathcal{E}}(1)\right)=h^{0}\left(\bar{X}, \bar{\varrho}^{*} \mathcal{O}_{\bar{Q}}(1)\right)=4 .
$$

Taking into account the isomorphism (4.2.4) we finally obtain $2 \leq \beta_{i} \leq 3$, and $\alpha_{j}=2$.

Let $1 \leq p \leq h$ be such that $\beta_{i}=2$ for $i=1, \ldots, p, \beta_{i}=3$ for $i=p+1, \ldots, h$. Comparing formulas (4.2.3) and (4.2.4), for any $t \geq 3$, we obtain $h=2 p$.

With the notations of the proof above, since $4 p \leq d-2$, it follows that if $d=4,5$ then $p=0$ necessarily. Hence we have the next result. See also Proposition 2.2 .

COROllary 4.3. Let $X$ and $\varrho$ be as in the above statement. If $d=4,5$ then $\mathcal{E} \cong \mathcal{O}_{Q}(2)^{\oplus d-2} \oplus \mathcal{O}_{Q}(4)$ and $\mathcal{F} \cong \mathcal{O}_{\mathcal{Q}}(\triangle)^{N}$, where $N=d(d-3) / 2$.

The results above are sharp as the following example shows.

EXAMPLE 4.4. Let $\mathcal{E}_{\tau}:=S(2)$ on a smooth quadric $Q \subseteq \mathbb{P}_{\mathbb{C}}^{4}$. Theorem 2.8 of [12] implies that $\mathcal{H}:=\mathcal{S}^{3} \mathcal{E}_{\tau} \otimes \operatorname{det} \mathcal{E}_{\tau}^{-1}$ is globally generated. We conclude that the general section $\eta \in H^{0}(Q, \mathcal{H})$ defines a cover $\tau: T \rightarrow Q$ of degree 3 with Tschirnhausen module $\mathcal{\mathcal { E }}_{\tau}$, where $T$ is smooth. (See [6, Theorem 3.6].)

Now let $\quad \mathcal{L}:=\omega_{T}^{-1} \otimes \tau^{*} \mathcal{O}_{Q}(1)$. We have that $\tau_{*} \mathcal{L} \cong \tau_{*}\left(\omega_{T \mid Q}^{-1} \otimes \tau^{*} \mathcal{O}_{Q}(4)\right)$. Duality for finite flat morphisms (see [9, Exercise III.6.10]) then yields $\tau_{*} \mathcal{L} \cong\left(\sigma_{*} \omega_{T \mid Q}^{2}\right)^{2} \otimes \mathcal{O}_{Q}(4)$.

Finally Proposition 5.1 of [6] implies that $\sigma_{*} \omega_{T \mid Q}^{2} \cong \mathcal{S}^{2} \mathcal{E}_{\tau}$. Then 


$$
\tau_{*} \mathcal{L} \cong\left(\mathcal{S}^{2} S(4)\right)^{\check{ } \otimes} \mathcal{O}_{Q}(4) \cong \mathcal{S}^{2} S(2),
$$

which is globally generated (again by Theorem 2.8 of [12]). Since $\tau$ is affine then the natural map $\tau^{*} \tau_{*} \mathcal{L} \rightarrow \mathcal{L}$ is surjective and therefore

$$
H^{0}(S, \mathcal{L}) \otimes \mathcal{O}_{S}=H^{0}\left(Q, \mathcal{S}^{2} S(2)\right) \otimes \mathcal{O}_{S} \rightarrow \tau^{*} \mathcal{S}^{2} S(2) \stackrel{\sim}{\longrightarrow} \tau^{*} \tau_{*} \mathcal{L} \rightarrow \mathcal{L}
$$

is surjective too. In particular $\mathcal{L}$ is globally generated. Let $\sigma: X \rightarrow T$ be the double cover associated to a general section of $\mathcal{L}^{2}$, so that $X$ is smooth. (For double covers see Section I.17 of [2].)

The map $\varrho:=\tau \circ \sigma: X \rightarrow Q$ is a cover of degree 6. Moreover

$$
\begin{aligned}
\varrho_{*} \mathcal{O}_{X} & =\tau_{*} \sigma_{*} \mathcal{O}_{X} \cong \tau_{*}\left(\mathcal{O}_{T} \oplus \mathcal{L}^{-1}\right) \cong \\
& \cong \mathcal{O}_{Q} \oplus \check{S}(-2) \oplus \tau_{*}\left(\omega_{S \mid Q} \otimes \tau^{*} \mathcal{O}_{Q}(-4)\right) \cong \\
& \cong \mathcal{O}_{Q} \oplus \check{S}(-2) \oplus\left(\left(\mathcal{O}_{Q} \otimes S(2)\right) \otimes \mathcal{O}_{Q}(-4)\right) \cong \\
& \cong \mathcal{O}_{Q} \oplus \check{S}(-2) \oplus \check{S}(-3) \oplus \mathcal{O}_{Q}(-4) .
\end{aligned}
$$

Thus the Tschirnhausen module $\breve{\mathcal{E}}$ of $\varrho$ satisfies $\mathcal{E} \cong S(2) \oplus S(3) \oplus \mathcal{O}_{Q}(4)$.

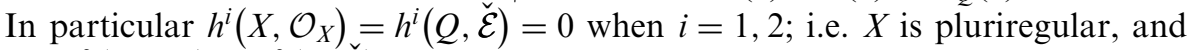
$p_{g}(S)=h^{3}\left(X, \mathcal{O}_{X}\right)=h^{3}(Q, \breve{\mathcal{E}})=5$. Finally notice that $\omega_{X} \cong \sigma^{*}\left(\omega_{T} \otimes \mathcal{L}\right) \cong \varrho^{*} \mathcal{O}_{Q}(1)$; i.e. $\varrho$ is the canonical map.

5. Covers of degree 4 of a smooth quadric. Let $\mathfrak{M}$ be the moduli space of canonically polarized, smooth threefolds with Hilbert polynomial

$$
h(t):=\chi\left(\omega_{X}^{t}\right)=\frac{1}{6} t(2 t-1)(t-1) d+8 t-4 .
$$

(See [17] for the existence of such an $\mathfrak{M}$.)

If $X$ is a smooth, pluriregular threefold of general type with $K_{X}^{3}=2 d$ and $p_{g}(X)=5$, then it represents a point $[X] \in \mathfrak{M}$. The set $\mathfrak{M}_{2 d, 5}$ of such points is open inside $\mathfrak{M}$. In particular $\mathfrak{M}_{2 d, 5}$ is a coarse moduli space for smooth pluriregular threefolds of general type with $K_{X}^{3}=2 d$ and $p_{g}(X)=5$.

We denote by $\mathfrak{M}_{2 d, 5}^{\text {quadric }}$ the locus in $\mathfrak{M}_{2 d, 5}$ of points representing threefolds $X$ whose canonical map $\varrho: X \rightarrow Q$ is a morphism onto a smooth quadric $Q \subseteq \mathbb{P}_{\mathbb{C}}^{4}$.

As has been previously noted, the invariants of such a cover are $\mathcal{E}=\mathcal{O}_{Q}(2) \oplus \mathcal{O}_{Q}(2) \oplus \mathcal{O}_{Q}(4)$ and $\mathcal{F}:=\mathcal{O}_{Q}(4)^{\oplus 2}$, and $X$ is the zero locus in $\mathbb{P}(\mathcal{E})$ of a section $\delta \in H^{0}\left(\mathbb{P}, \pi^{*} \breve{\mathcal{F}}(2)\right)$. Since, by the projection formula, $H^{0}\left(\mathbb{P}, \pi^{*} \breve{\mathcal{F}}(2)\right) \cong$ $H^{0}\left(Q, \mathcal{S}^{2} \mathcal{E} \otimes \check{\mathcal{F}}\right)$, they form a unirational family $F$ which is birationally isomorphic to $H^{0}\left(Q, \mathcal{S}^{2} \mathcal{E} \otimes \breve{\mathcal{F}}\right) / \operatorname{Aut}(\mathcal{F})$ and hence of dimension $h^{0}\left(Q, \mathcal{S}^{2} \mathcal{E} \otimes \breve{\mathcal{F}}\right)-\operatorname{dim}(\operatorname{Aut}(\mathcal{F}))$ $=168$, since $\operatorname{Aut}(\mathcal{F})=\mathrm{GL}_{2}$.

To compute $\operatorname{dim}\left(\mathfrak{M}_{8,5}^{\text {quadric }}\right)$, one has to take away the dimension of the group of automorphisms, which splits as $G:=\operatorname{Aut}(\mathcal{E}) / \mathbb{C}^{*} \ltimes \operatorname{Aut}(Q)$. Now $\operatorname{Aut}(Q)$ is 10-dimensional, whereas $\operatorname{Aut}(\mathcal{E})$ is formed by the order 3 non-degenerate square matrices 


$$
\left(\begin{array}{ccc}
a_{1} & a_{2} & 0 \\
a_{3} & a_{4} & 0 \\
b_{1} & b_{2} & a_{5}
\end{array}\right)
$$

where the $a_{i}^{\prime} s$ are constants and $b_{j} \in H^{0}\left(Q, \mathcal{O}_{Q}(2)\right)$. Hence $\operatorname{dim}(\operatorname{Aut}(\mathcal{E}))=32$. Finally the stabilizer in $G$ of each general point in $F$ is finite, since it is isomorphic to the automorphism group of the corresponding threefold of general type. Therefore

$$
\operatorname{dim}\left(\mathfrak{M}_{8,5}^{\text {quadric }}\right)=168-10-32=126 .
$$

Something more can be said about the component of $\mathfrak{M}_{8,5}$ to which those threefolds belong. In fact, the splitting of the bundle $\mathcal{E}$ is compatible with the bundle associated to a bidouble cover of the smooth quadric $Q$; i.e. a cover with an action of the group $\mathbb{Z}_{2} \times \mathbb{Z}_{2}$ on the fibers compatible with the cover. (See [7] or [13, par. 3]), branched on a divisor $B=2 D_{1}+2 D_{2}$, where $D_{i} \in H^{0}\left(Q, \mathcal{O}_{Q}(4)\right)$. In other words, the cover of degree 4 may be specialized to a bidouble cover.

Let $\bar{\varrho}: \bar{X} \rightarrow Q$ be such a cover: then one has the exact sequence of the normal sheaf to the map $\bar{Q}$, namely

$$
0 \rightarrow \Theta_{\bar{X}} \rightarrow \bar{\varrho}^{*} \Theta_{Q} \rightarrow \bigoplus_{i=1}^{2} \mathcal{O}_{D_{i}}(4) \oplus \mathcal{O}_{D_{i}}(2) \rightarrow 0,
$$

where $\Theta$ denotes the tangent sheaf. Recall that the above sequence induces a map $\partial: \bigoplus_{i=1}^{2} H^{0}\left(D_{i}, \mathcal{O}_{D_{i}}(4) \oplus \mathcal{O}_{D_{i}}(2)\right) \rightarrow H^{1}\left(\bar{X}, \Theta_{\bar{X}}\right)$ whose image $\operatorname{im}(\partial)$ locally parametrizes the family of the first order natural deformations of the bidouble cover in $\mathfrak{M}_{8,5}$. (For the definition of natural deformation see [7] or [13, par. 5].)

We denote by $\mathfrak{M}_{8,5}^{\text {natural }}$ the locus inside $\mathfrak{M}_{8,5}$ of points representing natural deformations of bidouble covers.

Proposition 5.3. $\mathfrak{M}_{8,5}^{\text {quadric }}$ is unirational of dimension 126 and it coincides with the family of natural deformations of bidouble covers of $Q$. The component of $\mathfrak{M}_{8,5}$ containing $\mathfrak{M}_{8,5}^{\text {quadric }}$ is unique and it has dimension 128.

Proof. By the projection formula $h^{i}\left(\bar{X}, \bar{\varrho}^{*} \Theta_{Q}\right)=h^{i}\left(Q, \Theta_{Q} \otimes \bar{\varrho}_{*} \mathcal{O}_{\bar{X}}\right)$. With the help of standard exact sequences it is not difficult to prove that

$$
h^{i}\left(\bar{X}, \bar{\varrho}^{*} \Theta_{Q}\right)= \begin{cases}10 & i=0 \\ 2 & i=1 \\ 0 & i \geq 2\end{cases}
$$

Moreover, since the $D_{i}$ 's are complete intersection surfaces in $\mathbb{P}_{\mathbb{C}}^{4}$,

$$
h^{i}\left(D_{i}, \mathcal{O}_{D_{i}}(4) \oplus \mathcal{O}_{D_{i}}(2)\right)= \begin{cases}54+14=68 & i=0 \\ 0 & i \geq 1\end{cases}
$$

Finally $H^{0}\left(\bar{X}, \Theta_{\bar{X}}\right)=0$, because $\bar{X}$ is of general type. On the one hand it follows that $\operatorname{im}(\partial)$ and hence $\mathfrak{M}_{8,5}^{\text {natural }}$ has dimension 126. On the other hand $\mathfrak{M}_{8,5}^{\text {natural }} \subseteq \mathfrak{M}_{8,5}^{\text {quadric }}$ has dimension 126 too, by (5.1). We conclude that they must coincide. 
The cohomology of the sequence (5.2) therefore gives

$$
h^{i}\left(\bar{X}, \Theta_{\bar{X}}\right)= \begin{cases}128 & i=1, \\ 0 & i \neq 1 .\end{cases}
$$

Since a neighborhood of $[\bar{X}]$ in $\mathfrak{M}_{8,5}$ is the quotient of the base of the Kuranishi family $B \cong H^{1}\left(\bar{X}, \Theta_{\bar{X}}\right)$ by $\operatorname{Aut}(\bar{X})$, it follows that $\mathfrak{M}_{8,5}^{\text {quadric }}$ is contained in a unique component of $\mathfrak{M}_{8,5}$.

It is known that the locus $\mathfrak{M}_{8,5}^{\text {birational }} \subseteq \mathfrak{M}_{8,5}$ of pluriregular threefolds $X$ with $K_{X}^{3}=8, p_{g}(X)=5$, having birational canonical morphism is irreducible of dimension 128. (See [15, Theorem 2.6].) The canonical images are hypersurfaces of degree 8 of $\mathbb{P}_{\mathbb{C}}^{4}$, with a surface of degree 8 as locus of double points; (see [15, Example 2.4]). The equation can be given as $f_{4} q^{2}+f_{2} \phi q-\phi^{2}=0$, where $f_{i}$ are general polynomials of degree $i$, for $i=2,4$, and $q, \phi$ are the generators of the ideal of the surface of double points, of degree 2 and 4 respectively.

One can observe that the graded canonical ring $\oplus_{m \in \mathbb{Z}} H^{0}\left(X, \omega_{X}^{m}\right)$ of such an $X$ has 5 generators in degree 1 , one generator in degree 2 and 2 relations in degree 4 (Riemann-Roch for the plurigenera). This means that $X$ may be thought of as the complete intersection of two hypersurfaces of degree 4 in the weighted projective space $\mathbb{P}(1,1,1,1,1,2)$.

Let $x_{0}, \ldots, x_{4}$ be a system of generators of degree 1 and let $y$ be a generator of degree 2 for the canonical ring of $X$. One can suppose, without loss of generality, that the equations defining $X$ in $\mathbb{P}(1,1,1,1,1,2)$ (or equivalently, in $\mathbb{P}\left(\mathcal{O}_{\mathbb{P}_{\mathbb{C}}^{4}} \oplus \mathcal{O}_{\mathbb{P}_{\mathbb{C}}^{4}}(2)\right.$ ); in which case $y$ is thought of as an affine variable), are

$$
y^{2}+\alpha_{1}(x) y+\beta_{1}(x)=y^{2}+\alpha_{2}(x) y+\beta_{2}(x)=0,
$$

where $\alpha_{h} \in H^{0}\left(\mathbb{P}_{\mathbb{C}}^{4}, \mathcal{O}_{\mathbb{P}_{\mathbb{C}}^{4}}(2)\right)$ and $\beta_{h} \in H^{0}\left(\mathbb{P}_{\mathbb{C}}^{4}, \mathcal{O}_{\mathbb{P}_{\mathbb{C}}^{4}}(4)\right)$. The equation of $X \subseteq \mathbb{P}_{\mathbb{C}}^{4}$ given above can be recovered by eliminating the $y$ coordinate.

Proposition 5.4. For each threefold $X$ representing a point in $\mathfrak{M}_{8,5}^{q u a d r i c}$ there exists a 1-parameter family whose general point lies in $\mathfrak{M}_{8,5}^{\text {birational }}$.

Proof. Consider a threefold $X$ in $\mathfrak{M}_{8,5}^{q u a d r i c}$ and its equations $(2.2 .1)$ in $\mathbb{P}:=\mathbb{P}(\mathcal{E})$. We can embed $\mathbb{P} \subseteq \overline{\mathbb{P}}:=\mathbb{P}\left(\mathcal{O}_{\mathbb{P}_{\mathbb{C}}^{4}} \oplus \mathcal{O}_{\mathbb{P}_{\mathbb{C}}^{4}} \oplus \mathcal{O}_{\mathbb{P}_{\mathbb{C}}^{4}}(2)\right)$.

With this in mind, up to a suitable linear transformation of $u_{1}, u_{2}, u_{3}$, we can assume that the equations of $X$ in the open subset $U:=\left\{u_{3} \neq 0\right\} \subseteq \overline{\mathbb{P}}$ are

$$
y_{1}^{2}+a_{1}(x) y_{2}+b_{1}(x)=y_{2}^{2}+a_{2}(x) y_{2}+b_{2}(x)=q(x)=0,
$$

where $y_{i}=u_{i} / u_{3}, a_{h} \in H^{0}\left(\mathbb{P}_{\mathbb{C}}^{4}, \mathcal{O}_{\mathbb{P}_{\mathbb{C}}^{4}}(2)\right), b_{h} \in H^{0}\left(\mathbb{P}_{\mathbb{C}}^{4}, \mathcal{O}_{\mathbb{P}_{\mathbb{C}}^{4}}(4)\right)$ and $Q=\{q=0\}$.

Consider $X_{t} \subseteq \overline{\mathbb{P}}$ given by

$$
y_{1}^{2}+a_{1}(x) y_{2}+b_{1}(x)=y_{2}^{2}+a_{2}(x) y_{2}+b_{2}(x)=t y_{2}+q(x)=0 .
$$

(Notice that $\left\{t y_{2}+q(x)=0\right\} \cong \mathbb{P}\left(\mathcal{O}_{\mathbb{P}_{\mathbb{C}}^{4}} \oplus \mathcal{O}_{\mathbb{P}_{\mathbb{C}}^{4}}(2)\right)$.) Then $X_{0} \cong X$, whereas if $t \neq 0$ we can eliminate $y_{2}$ from the third equation, thus obtaining a general threefold in $\mathfrak{M}_{8,5}^{\text {birational }}$. 
COROLlaRY 5.5. The closure of $\mathfrak{M}_{8,5}^{\text {birational }}$ in $\mathfrak{M}_{8,5}$ is the unique component containing $\mathfrak{M}_{8,5}^{\text {quadric }}$.

Proof. It is an immediate consequence of Propositions 5.3 and 5.4.

REMARK 5.6. Consider the locus $\mathfrak{M}_{8,5}^{\text {quartic }} \subseteq \mathfrak{M}_{8,5}$ of points representing threefolds $X$ whose canonical map is a finite morphism of degree 2 onto a smooth quartic $F \subseteq \mathbb{P}_{\mathbb{C}}^{4}$. Then $\operatorname{dim}\left(\mathfrak{M}_{8,5}^{\text {quartic }}\right)=113$ and, as above, it can be proved that the unique component of $\mathfrak{M}_{8,5}$ is again the closure of $\mathfrak{M}_{8,5}^{\text {birational }}$. (See Remark 2.2 of [14].)

6. Covers of degree 5,6 of a smooth quadric. In this section we give some information about the dimension of $\mathfrak{M}_{2 d, 5}^{\text {quadric }}$, when $d=5,6$.

6.1. $d=5$. Each cover of degree 5 of a smooth quadric of $\mathbb{P}_{\mathbb{C}}^{4}$ is the pfaffian locus in $\mathbb{P}(\mathcal{E})$ of a section $\delta \in H^{0}\left(\mathbb{P}, \pi^{*} \Lambda^{2} \mathcal{F} \otimes \pi^{*} \operatorname{det} \mathcal{E}^{-1}(1)\right)$, where $\mathcal{E}:=\mathcal{O}_{Q}(2) \oplus$ $\mathcal{O}_{Q}(2) \oplus \mathcal{O}_{Q}(2) \oplus \mathcal{O}_{Q}(4)$ and $\mathcal{F}:=\mathcal{O}_{Q}(4)^{\oplus 5}$.

Since, by the projection formula, $H^{0}\left(H^{0}\left(\mathbb{P}, \pi^{*} \Lambda^{2} \mathcal{F} \otimes \pi^{*} \operatorname{det} \mathcal{E}^{-1}(1)\right) \cong\right.$ $H^{0}\left(Q, \Lambda^{2} \mathcal{F} \otimes \operatorname{det} \mathcal{E}^{-1} \otimes \mathcal{E}\right)$, they form a unirational family given by $H^{0}\left(Q, \Lambda^{2} \mathcal{F} \otimes \mathcal{E} \otimes \operatorname{det} \mathcal{E}^{-1}\right) / \operatorname{Aut}(\mathcal{F})$, which is thus of dimension

$$
h^{0}\left(Q, \Lambda^{2} \mathcal{F} \otimes \mathcal{E} \otimes \operatorname{det} \mathcal{E}^{-1}\right)-\operatorname{dim}(\operatorname{Aut}(\mathcal{F}))=170-25=145,
$$

since $\operatorname{Aut}(\mathcal{F})=\mathrm{GL}_{5}$.

Proposition 6.1.1. $\mathfrak{M}_{10,5}^{q u a d r i c}$ is unirational of dimension 84.

Proof. Again to compute $\operatorname{dim}\left(\mathfrak{M}_{10,5}^{\text {quadric }}\right)$, one has to take away the dimension of the group of automorphisms $\operatorname{Aut}(\mathcal{E}) / \mathbb{C}^{*} \ltimes \operatorname{Aut}(Q)$. Now $\operatorname{Aut}(\mathcal{E})$ is formed by the order 4 non-degenerate square matrices

$$
\left(\begin{array}{cccc}
a_{1} & a_{2} & a_{3} & 0 \\
a_{4} & a_{5} & a_{6} & 0 \\
a_{7} & a_{8} & a_{9} & 0 \\
b_{1} & b_{2} & b_{3} & a_{10}
\end{array}\right),
$$

where the $a_{i}^{\prime} s$ are constants and $b_{j} \in H^{0}\left(Q, \mathcal{O}_{Q}(2)\right)$. Hence $\operatorname{dim}(\operatorname{Aut}(\mathcal{E}))=52$, and the stabilizer in $G$ of each general point in $F$ must be finite. (It is isomorphic to the automorphism group of the corresponding threefold.)

Notice that the locus $\mathfrak{M}_{10,5}^{\text {birational }}$ of points of $\mathfrak{M}_{10,5}$ representing threefolds whose canonical map is birational has dimension 87. (See Theorem 4.5 of [15].)

Unfortunately such covers cannot be abelian. Thus we cannot compute the dimension of the component of $\mathfrak{M}_{10,5}$ of smooth, pluriregular threefolds of general type $X$ with $K_{X}^{3}=10, p_{g}(X)=5$ containing them via the method used in the previous section for $\mathfrak{M}_{8,5}^{\text {quadric }}$.

6.2. $d=6$. Now consider the case $K_{X}^{3}=12$. Each scandinavian cover of a smooth quadric of $\mathbb{P}_{\mathbb{C}}^{4}$ is the degeneracy locus $D_{1}(\delta) \subseteq \mathbb{P}:=\mathbb{P}(\mathcal{E})$ of a section $\delta \in H^{0}\left(\mathbb{P}, \pi^{*} \check{\mathcal{A}} \otimes \pi^{*} \mathcal{B}(1)\right) \cong \operatorname{hom}_{\mathcal{O}_{\mathbb{P}}}\left(\pi^{*} \mathcal{A}, \pi^{*} \mathcal{B}(1)\right), \quad$ where $\quad \mathcal{E}:=\mathcal{O}_{Q}(2)^{\oplus 4} \oplus \mathcal{O}_{Q}(4)$, $\mathcal{A}:=\mathcal{O}_{Q}^{\oplus 3}, \mathcal{B}:=\mathcal{O}_{\mathbb{P}_{\mathbb{C}}^{4}}(-2)^{\oplus 3}$. 
Notice that $g:=(a, b) \in G:=\operatorname{Aut}(\mathcal{A}) \times \operatorname{Aut}(\mathcal{B})$ acts on the above $\delta$ by $g(\delta)=b \delta^{t} a$. Hence the action of $G$ has generic stabilizer $G_{\delta}:=\left\{\left(\lambda I_{3}, \lambda^{-1} I_{3}\right) \mid \lambda \in \mathbb{C}^{*}\right\}$.

Since $H^{0}\left(\mathbb{P}, \pi^{*} \breve{\mathcal{A}} \otimes \pi^{*} \mathcal{B}(1)\right) \cong H^{0}(Q, \breve{A} \otimes \mathcal{B} \otimes \mathcal{E})$, scandinavian covers form a unirational family $F:=H^{0}(Q, \mathcal{A} \otimes \mathcal{B} \otimes \mathcal{E}) /(\operatorname{Aut}(\mathcal{A}) \times \operatorname{Aut}(\mathcal{B}))$, whose dimension is $\operatorname{dim}(F)=h^{0}(Q, \mathcal{A} \otimes \mathcal{B} \otimes \mathcal{E})-\operatorname{dim}(\operatorname{Aut}(\mathcal{A}))-\operatorname{dim}(\operatorname{Aut}(\mathcal{B}))+1=162-17=145$, since $\operatorname{Aut}(\mathcal{A})=\operatorname{Aut}(\mathcal{B})=\mathrm{GL}_{3}$.

Proposition 6.2.1. $\operatorname{dim}\left(\mathfrak{M}_{12,5}^{\text {quadric }}\right) \geq 63$.

Proof. $\mathfrak{M}_{12,5}^{\text {quadric }}$ contains the locus $\mathfrak{M}_{12,5}^{\text {scandinavian }}$ of threefolds whose canonical map is a scandinavian cover of a smooth quadric. (See the description above.) For computing $\operatorname{dim}\left(\mathfrak{M}_{12,5}^{\text {scandinavian }}\right)$ one has to take away the dimension of the group of automorphisms $\operatorname{Aut}(\mathcal{E}) / \mathbb{C}^{*} \ltimes \operatorname{Aut}(Q)$. Now $\operatorname{Aut}(\mathcal{E})$ is formed by the order 5 nondegenerate square matrices

$$
\left(\begin{array}{ccccc}
a_{1} & a_{2} & a_{3} & a_{4} & 0 \\
a_{5} & a_{6} & a_{7} & a_{8} & 0 \\
a_{9} & a_{10} & a_{11} & a_{12} & 0 \\
a_{13} & a_{14} & a_{15} & a_{16} & 0 \\
b_{1} & b_{2} & b_{3} & b_{4} & a_{17}
\end{array}\right),
$$

where the $a_{i}^{\prime} s$ are constants and $b_{j} \in H^{0}\left(Q, \mathcal{O}_{Q}(2)\right)$. Hence $\operatorname{dim}(\operatorname{Aut}(\mathcal{E}))=73$. The stabilizer in $G$ of each general point in $F$ is finite.

Notice that the locus $\mathfrak{M}_{12,5}^{\text {birational }}$ of points of $\mathfrak{M}_{12,5}$ representing threefolds whose canonical map is birational has dimension 67. (See Theorem 5.4 of [15]: the correct value is 67 as it follows from the proof.) It could be interesting to check that $\operatorname{dim}\left(\mathfrak{M}_{12,5}^{\text {quadric }}\right)=\operatorname{dim}\left(\mathfrak{M}_{12,5}^{\text {scandinavian }}\right)$. If this is the case

$$
\operatorname{dim}\left(\mathfrak{M}_{2 d, 5}^{\text {birational }}\right)=\operatorname{dim}\left(\mathfrak{M}_{2 d, 5}^{\text {quadric }}\right)+d-2, \quad d=3,4,5,6 .
$$

Notice that Example 4.4 assures that $\mathfrak{M}_{12,5}^{\text {scandinavian }} \neq \mathfrak{M}_{12,5}^{\text {quadric }}$.

7. Examples of pluriregular threefolds with odd $p_{g} \geq 7$. Let $X$ be a smooth threefold. Assume that $X$ is pluriregular, with $K_{X}^{3}=d\left(p_{g}(X)-3\right)$ for $p_{g}(X) \geq 6$. Moreover we consider only the case in which $\left|K_{X}\right|$ has no fixed part and the canonical map $\varphi_{X}: X \rightarrow \mathbb{P}_{\mathbb{C}}^{p_{g}(X)-1}$ is finite of degree $d$. (Hence $\left|K_{X}\right|$ is base-point-free.)

It follows that $W:=\varphi_{X}(X) \subseteq \mathbb{P}_{\mathbb{C}}^{p_{g}(X)-1}$ has minimal degree $p_{g}(X)-3$. Hence it is a rational normal scroll. Let $\widetilde{W}$ be a minimal resolution of the singularities of $W$. There are integers $0 \leq a_{1} \leq a_{2} \leq a_{3}$ such that

$$
\tilde{W}=\mathbb{P}\left(\mathcal{O}_{\mathbb{P}_{\mathbb{C}}^{1}}\left(a_{1}\right) \oplus \mathcal{O}_{\mathbb{P}_{\mathbb{C}}^{1}}\left(a_{2}\right) \oplus \mathcal{O}_{\mathbb{P}_{\mathbb{C}}^{1}}\left(a_{3}\right)\right) \stackrel{j}{\longrightarrow} W \subseteq \mathbb{P}_{\mathbb{C}}^{p_{g}(X)-1} .
$$

The triple $\left(a_{1}, a_{2}, a_{3}\right)$ is called the type of the scroll $W$ and we write $W_{\left(a_{1}, a_{2}, a_{3}\right)}$ instead of $W$. If $a_{1}>0$ then $W=\widetilde{W}$. Otherwise $W$ has at most rational singularities.

In what follows we shall assume that $W$ is smooth. The embedding $W \subseteq \mathbb{P}_{\mathbb{C}}^{p_{g}(X)-1}$ is induced by the tautological bundle $\mathcal{O}_{W}(1)$; hence $p_{g}(X)-1=a_{1}+a_{2}+a_{3}+2$. In this case the canonical map is a cover $\varrho: X \rightarrow W$ of degree $d$. 
Recall that $\operatorname{Pic}(W)=\mathbb{Z} \mathcal{O}_{W}(1) \oplus \mathbb{Z} p^{*} \mathcal{O}_{\mathbb{P}_{\mathbb{C}}^{1}}(1)$, where $p: W \rightarrow \mathbb{P}_{\mathbb{C}}^{1}$ is the natural projection.

THEOREM 7.1. For each positive integer $v \geq 2$, each $d=4,5,6$ and each triple $1<a_{1} \leq a_{2} \leq a_{3}$ with $a_{1}+a_{2}+a_{3}=2 v, 3 a_{1}+1 \geq a_{2}+a_{3}$, there exist smooth, pluriregular, threefolds of general type $X$ with $p_{g}(X)=2 v+3, K_{X}^{3}=2 v d$, whose canonical map are covers $\varrho: X \rightarrow W_{\left(a_{1}, a_{2}, a_{3}\right)}$ of degree $d$.

Proof. Fix $n, d$ and $\left(a_{1}, a_{2}, a_{3}\right)$ as above and define $2 v:=a_{1}+a_{2}+a_{3}$. The invertible sheaf $\mathcal{L}:=\mathcal{O}_{W}(2) \otimes p^{*} \mathcal{O}_{\mathbb{P}_{C}^{1}}(1-v)$ is globally generated if $2 a_{1} \geq v-1$; i.e. if $3 a_{1}+1 \geq a_{2}+a_{3}$. Hence we can apply Propositions 3.1, 3.2 and 3.3 above and we get covers $\varrho: X \rightarrow W$ of degree $d$ with $X$ smooth. Moreover

$$
h^{i}\left(X, \mathcal{O}_{X}\right)=h^{i}\left(W, \varrho_{*} \mathcal{O}_{X}\right)=h^{i}\left(W, \mathcal{O}_{W}\right)+h^{i}(W, \check{\mathcal{E}}),
$$

where $\mathcal{E}:=\mathcal{L}^{\oplus d-2} \oplus \mathcal{L}^{2}$. Since $W$ is pluriregular and $h^{i}\left(W, \mathcal{L}^{-1}\right)=h^{i}\left(W, \mathcal{L}^{-2}\right)=0$ for $i=1,2$, each such $X$ is pluriregular too.

Finally $\omega_{W}=\mathcal{O}_{W}(-3) \otimes p^{*} \mathcal{O}_{\mathbb{P}_{\mathbb{C}}^{1}}(2 v-2)$. Hence

$$
\omega_{X}=\mathcal{O}_{W}(4) \otimes p^{*} \mathcal{O}_{\mathbb{P}_{\mathbb{C}}^{1}}(2-2 v) \otimes \varrho^{*} \omega_{W}=\mathcal{O}_{W}(1)=\varphi_{X}^{*} \mathcal{O}_{\mathbb{P}_{\mathbb{C}}^{n-1}}
$$

It follows that $p_{g}(X)=n$ and $\varrho$ is the canonical map of $X$ : in particular $K_{X}^{3}=2 v d$.

As an example, we now compute the number of moduli in the case $K_{X}^{3}=8 v$, $v \geq 2$. Recall that $\mathcal{L}:=\mathcal{O}_{W}(2) \otimes p^{*} \mathcal{O}_{\mathbb{P}_{\mathbb{C}}^{1}}(1-v)$ where $2 v:=a_{1}+a_{2}+a_{3}$.

LeMma 7.2. Let $W \subset \mathbb{P}_{\mathbb{C}}^{2 v+2}$ be a nonsingular scroll of degree $2 v$. We have

$$
\begin{aligned}
h^{0}(W, \mathcal{L}) & =2 v+12+h^{1}(W, \mathcal{L}), \\
h^{0}\left(W, \mathcal{L}^{2}\right) & =10 v+45+h^{1}\left(W, \mathcal{L}^{2}\right), \\
h^{2}\left(W, \mathcal{L}^{m}\right) & =h^{3}\left(W, \mathcal{L}^{m}\right)=0, \quad \text { for } m=1,2 .
\end{aligned}
$$

Proof. Consider the exact sequence

$$
0 \rightarrow \mathcal{O}_{W}(2 m) \otimes p^{*} \mathcal{O}_{\mathbb{P}_{\mathbb{C}}^{1}}(m(1-v)) \rightarrow \mathcal{O}_{W}(2 m) \rightarrow \mathcal{O}_{D_{m}}(2 m) \rightarrow 0,
$$

where $D_{m} \in\left|p^{*} \mathcal{O}_{\mathbb{P}_{\mathbb{C}}^{1}}(m(1-v))\right|$. Owing to the smoothness of $W$ each such general $D_{m}$ is formed by $m(v-1)$ disjoint planes.

Since the scrolls are projectively Cohen-Macaulay, the dimension of the linear system cut by the hypersurfaces of the projective space depends only on the degree. Therefore, we compute $h^{0}\left(W, \mathcal{O}_{W}(2 m)\right)$ on a particular scroll by means of the projection $p$ on $\mathbb{P}_{\mathbb{C}}^{1}$. One has

$$
h^{0}\left(W, \mathcal{O}_{W}(2 m)\right)=h^{0}\left(\mathbb{P}_{\mathbb{C}}^{1}, \mathcal{S}^{2 m}\left(\mathcal{O}_{\mathbb{P}_{\mathbb{C}}^{1}}\left(a_{1}\right) \oplus \mathcal{O}_{\mathbb{P}_{\mathbb{C}}^{1}}\left(a_{2}\right) \oplus \mathcal{O}_{\mathbb{P}_{\mathbb{C}}^{1}}\left(a_{3}\right)\right)\right)
$$

The computation in the 3 cases $(a, a, a),(a, a, a+1),(a, a+1, a+1)$, depending on the class of $2 v$ modulo 3 (those are called balanced scrolls), by projecting on $\mathbb{P}_{\mathbb{C}}^{1}$ gives $h^{0}\left(W, \mathcal{O}_{W}(2)\right)=8 v+6$ and $h^{0}\left(W, \mathcal{O}_{W}(4)\right)=40 v+15$. 
Moreover, $h^{0}\left(D_{1}, \mathcal{O}_{D_{1}}(2)\right)=6(v-1)$ and $h^{0}\left(D_{2}, \mathcal{O}_{D_{2}}(4 H)\right)=30(v-1)$. Since one has $h^{i}\left(W, \mathcal{O}_{W}(2 m)\right)=0$, for $m \geq 1$, it follows from the exact sequence of restriction that

$$
h^{0}\left(W, \mathcal{L}^{m}\right)=h^{0}\left(W, \mathcal{O}_{W}(2 m)\right)-h^{0}\left(D_{m}, \mathcal{O}_{D_{m}}(2 m)\right)+h^{1}\left(W, \mathcal{L}^{m}\right) .
$$

Finally for $m \geq 1$ one has $h^{i}\left(D_{m}, \mathcal{O}_{D_{m}}(2 m)\right)=0$.

Consider the balanced case when $\left(a_{1}, a_{2}, a_{3}\right)$ coincides with either $(a, a, a)$ or $(a, a, a+1)$ or $(a, a+1, a+1)$ according to the class of $2 v$ modulo 3 . Since $h^{1}\left(W, \mathcal{L}^{m}\right)=0, m=1,2$, we obtain the family $F:=H^{0}\left(\mathbb{P}, \pi^{*} \breve{\mathcal{F}}(2)\right) / \operatorname{Aut}(\mathcal{F})$ of dimension

$$
h^{0}\left(\mathbb{P}, \pi^{*} \breve{\mathcal{F}}(2)\right)-\operatorname{dim}(\operatorname{Aut}(\mathcal{F}))=h^{0}\left(W, \check{\mathcal{F}} \otimes \mathcal{S}^{2} \mathcal{E}\right)-\operatorname{dim}\left(\mathrm{GL}_{2}\right)=28 v+140
$$

The corresponding moduli spaces $\mathfrak{M}_{8 v, 2 v+3, \mathcal{E}}^{\text {scroll }}$ of isomorphism classes of nonsingular threefolds with $p_{g}=2 v+3, K_{X}^{3}=8 v$ and canonical map of degree 4 with Tschirnhausen module $\mathcal{E}$ onto a smooth scroll $W \subseteq \mathbb{P}_{\mathbb{C}}^{2 v+2}$ is obtained by quotienting out the automorphism group of $\mathbb{P}$ which is $G=\operatorname{Aut}(\mathcal{E}) / \mathbb{C}^{*} \ltimes \operatorname{Aut}(W)$. As above the stabilizer in $G$ of each general point in $F$ is finite, since it corresponds to the automorphism group of the corresponding threefold of general type.

We have $\operatorname{dim}(\operatorname{Aut}(\mathcal{E}))=5+2 h^{0}(W, \mathcal{L})=4 v+28$. Moreover $\operatorname{Aut}(W)=$ $\operatorname{Aut}_{\mathbb{P}_{\mathbb{C}}^{1}}(W) \ltimes \operatorname{Aut}\left(\mathbb{P}_{\mathbb{C}}^{1}\right)$. $\left(\operatorname{Aut}_{\mathbb{P}_{\mathbb{C}}^{1}}(W)\right.$ is the group of the automorphisms of $W$ fixing the fibers of $p$.) Thus in the balanced case one always has $\operatorname{dim}(\operatorname{Aut}(W))=11$. Therefore we have a family of threefolds, up to isomorphism, of dimension $24 v+101$.

We remark that this number is the biggest we can obtain with the above construction, since $h^{0}\left(W, \breve{\mathcal{F}} \otimes \mathcal{S}^{2} \mathcal{E}\right)$ decreases while $\operatorname{dim}\left(\operatorname{Aut}_{\mathbb{P}_{\mathbb{C}}^{1}}(W)\right)$ increases for unbalanced scrolls. Therefore we have the following result.

Proposition 7.3. $\mathfrak{M}_{8 v, 2 v+3, \mathcal{E}}^{\text {scroll }}$ is unirational of dimension $24 v+101$ for $v \geq 2$.

\section{REFERENCES}

1. V. Ancona and G. Ottaviani, Some applications of Beilinson's theorem to projective spaces and quadrics, Forum Math. 3 (1991), 157-176. 1984).

2. W. Barth, C. Peters and A. Van de Ven, Compact complex surfaces (Springer-Verlag,

3. D.A. Buchsbaum and D. Eisenbud, Algebra structures for finite free resolutions, and some structure theorems for ideals of codimension 3, Amer. J. Math. 99 (1977), 447-485.

4. G. Casnati, Covers of algebraic varieties II. Covers of degree 5 and construction of surfaces, J. Algebraic Geom. 5 (1996), 461-477.

5. G. Casnati, Cover of algebraic varieties IV. A Bertini theorem for scandinavian covers of degree 6, Forum Math., 13 (2001), 21-36.

6. G. Casnati and T. Ekedahl, Covers of algebraic varieties I. A general structure theorem, covers of degree 3, 4 and Enriques surfaces, J. Algebraic Geom. 5 (1996), 439-460.

7. F. Catanese, On the moduli spaces of surfaces of general type, J. Differential Geom. 19 (1984), 483-515.

8. T.H. Gulliksen and O.G. Negård, Un complex résolvant pour certains ideaux determinantiels, Comp. Rend. Acad. Sci. Paris Ser. A 274 (1972), 16-18.

9. R. Hartshorne, Algebraic geometry (Springer-Verlag, 1977).

10. Horikawa, E., Algebraic surfaces of general type with small $c_{1}^{2}$ I, Ann. Math 104 (1976), 357-387. II. Invent. Math. 37 (1976), 121-155. III. Invent. Math. 47 (1978), 209-248. IV. Invent. Math. 50 (1979), 103-128. 
11. R. Miranda, Triple covers in algebraic geometry, Amer. J. Math. 107 (1985), 11231158.

12. G. Ottaviani, Spinor bundles on quadrics, Trans. Amer. Math. Soc. 307 (1988), 301316.

13. R. Pardini, Abelian covers of algebraic varieties, J. Reine. Angew. Math. 417 (1991), $191-213$

14. P. Supino, Triple covers of 3 -folds as canonical maps, Comm. Algebra 26 (1998), $1457-1487$.

15. P. Supino, On canonical hypersurfaces of $\mathbb{P}^{4}$, Math. Z. 231 (1999), 489-516.

16. P. Supino, On threefolds with $K^{3}=2 p_{g}-6$ (to appear).

17. E. Viehweg, Weak positivity of certain Hilbert points II, Invent. Math. 101 (1990), $191-223$. 